\title{
A Modelling and Tabu Search Heuristic for a Continuous Galvanizing Line Scheduling Problem
}

\author{
Lixin TANG and Cong GAO \\ Liaoning Key Laboratory of Manufacturing System and Logistics, The Logistics Institute, Northeastern University, Shenyang, \\ Liaoning Province, 110004, P. R. China. E-mail: lixintang@mail.neu.edu.cn
}

(Received on June 25, 2008; accepted on December 24, 2008)

\begin{abstract}
In this paper, we address a continuous galvanizing line scheduling problem in a steel plant. The continuous galvanizing line we research produces principally two kinds of coils, inner galvanized coils and outer galvanized coils. Due to the technical constraints, outer coils can not be produced continuously more than a specified number and some inner coils must be inserted between outer coils. The problem is to find a schedule of all coils that minimizes the sum of changeover costs, the number of inserted inner coils and the number of transition coils used. The difficulty of solving the problem lies in the interrelation of sequencing these two kinds of coils. We formulate the scheduling problem as an integer programming model by considering above practical requirements. A heuristic based on tabu search is developed to solve the problem. The matching algorithm is used during the procedure of generating an initial solution where the optimal assignment of candidate inner coils to be inserted and positions is found by Hungarian method and then the saving step is used to reduce the number of inner coils inserted. In implementing the tabu search heuristic, intensification search, diversification search and path relinking are used to improve the effectiveness of the algorithm. 28 instances of four sizes are randomly generated to simulate the actual production data. For all the instances with 20 and 30 coils and four out of five instances with 50 coils, the tabu search heuristic finds the optimal schedules as CPLEX does. For other instances, the heuristic always obtains the better schedules and consumes much less computation time than CPLEX. In real production environment, hundreds of coils are needed to be scheduled, and the heuristic is able to generate high quality schedules within reasonable computation time.
\end{abstract}

KEY WORDS: continuous galvanizing line; integer programming model; tabu search.

\section{Introduction}

With good mechanical properties and corrosion-resistance, galvanized coils are used in many fields nowadays, such as auto industry and household appliance industry. Because of high additional value of galvanized product, steel plants pay much more attention on how to make continuous galvanizing lines (CGL) run efficiently. In this paper, we address a scheduling problem of continuous galvanizing line, which aims to reduce production cost and improve production efficiency.

A steel plant usually consists of two parts, the primary steel making part and the finishing steel making part. In primary steel making part, slabs are rolled into hot coils in hot strip mills. Sequentially hot coils are transported to cold mills, where hot coils are shaped thinner and produced into cold coils. Cold coils have bad mechanical properties and are prone to rusting. In order to meet advanced requirement, it is necessary to process cold coils by annealing and galvanizing. These two processes are integrated into CGL. Coils are heated and cooled in a continuous annealing furnace to improve the mechanical properties. And zinc coats are coated on surfaces of coils to protect them from corrosion.
The CGL we research in the paper produces principally coils used to make autos. The outer surfaces of autos are made of outer galvanized coils which have very high surface finish requirement, and the inner surfaces are made of inner galvanized coils with normal surface finish requirement. Because of very high requirement of outer coils on surface finish, it is required that outer coils must be produced at the beginning of schedules when rollers are smooth and the number of outer coils produced continuously can not exceed a specified number. In our best knowledge, no published paper has described or solved such a similar problem so far. The interrelation between scheduling two kinds of coils makes the problem difficult to solve.

In this paper, we propose a heuristic based on tabu search. The procedure of generating initial solutions is divided into three subroutines, and Hungarian method which is the classic method to solve assignment problems are employed in one of subroutines. Five neighborhoods are designed in tabu search phase. Finally to improve the effectiveness of the heuristic, three additional strategies, intensification search, diversification search and path relinking, are used in the heuristic.

Many researches have been published on scheduling in steel industry. Lee et al. ${ }^{1)}$ described a scheduling problem 
in primary production which aimed to minimize production cost and delivery on time. For the problem, several approaches are proposed. Lopez et al. ${ }^{2)}$ modeled hot strip mill production scheduling problem as the Prize Collecting Traveling Salesman Problem and designed a heuristic method based on tabu search to obtain good schedules. Cowling el al. ${ }^{3)}$ addressed also hot strip mill and focused on dynamic scheduling. Cowling ${ }^{4)}$ presented a flexible decision support system for hot strip mills where a heuristic based on tabu search was embedded to solve the scheduling problem. Tang et al. ${ }^{5)}$ reviewed planning and scheduling systems and methods for integrated steel production. Most researches addressed primary steel making, such as continuous casting and hot rolling, but few research paid attention on finishing steel making before. In recent years, more and more researchers have focused on finishing steel making. Moon and Hrymak $^{6}$ addressed a scheduling problem for the batch annealing furnace which process steel coils in the heat treatment way and proposed a novel algorithm to solved the problem. A reactive method for color coating production in steel industry was developed by Tang and Wang, ${ }^{7)}$ which meets the fact that originally established schedules often need to be adjusted.

Two papers are about the scheduling of CGL. Okano et $a l .{ }^{8)}$ solved a continuous galvanizing line scheduling problem, where a schedule should be fish-shaped. They explicitly considered six conflicting objectives and formulated the problem with a mathematical model. The CGL can be idle to wait for an unreleased cold coil, which distinguishes from other machines in steel industry. A local search based on three operations was employed to improve the initial solution. Distances among coils were defined, which helps to exclude unpromising operations during the addition heuristic and the local search. They claimed that the heuristic could obtain better solutions than most of the example solutions.

Verdejo et al. ${ }^{9)}$ presented a method based on TS to solve CGLSP. In Ref. 9), coils were categorized into three types, and the sequence among the three types was fixed. The paper dealt with scheduling coils of only one of these three types. There was no interrelation among different types of coils. The concept, fitness level, was introduced and tabu search algorithm was implemented in Ref. 9). The algorithm allowed searching the domain of unfeasible solution, and a set named CONFLICT was used to keep the violation pairs of adjacent coils. The neighborhood was constructed with the solutions obtained by the moves which relocated coils between pairs in CONFILCT. If CONFILT was empty, the set was extended with the pairs whose fitness are not in the best status. The simple neighborhood in Ref. 9) suited the problem very much because of the character that the suitability between coils was scaled by the fitness which is a discrete value. In most other research, including ours, the suitability is scaled by a continuous value.

Though we also employ TS to solve the scheduling problem of CGL, three principally differences lie between two researches. Firstly, the characters of two scheduling problems are distinguishing. In our problem, outer coils must be sequenced with inner coil insertion, and thus the sequencing of outer coils and inner coils affect each other. Secondly, we construct the model describing the special sched- uling problem. Finally, the heuristic based on TS is of much difference to the algorithm in Ref. 9). The procedure of generating an initial solution is based on the match idea, and Hungarian method is used during the procedure. Our tabu search heuristic searches a composite neighborhood of five individual neighborhoods during local search, and the quadrate tabu list is used to keep the tabu moves. Due to the characters of the problem, the totally different diversification search is implemented in our heuristic, which focuses on the scheduling of outer coils and repair measures are performed to infeasible schedules resulted from diversification search.

\section{Problem Description}

To illustrate the objectives and the constraints clearly, we first describe the production process flow of CGL production. The CGL processes coils in a continuous way with annealing, galvanizing and surface post-processing. Before annealing, the individual cold coils from cold mills need to be welded into steel strips on the welding machine to satisfy continuous processing requirement. In the continuous annealing furnace, coils are heated and cooled in accordance with annealing temperature curves. Annealed coils go through the zinc pot where coils' surfaces are galvanized with zinc coats whose thickness depends on customer order requirement and the coats are solidified in the alloy furnace. Finally surface post-processing is performed before the process finishes.

The CGL produces two kinds of galvanized coils, inner coils and outer coils. Because outer coils have higher surface requirement, while the rollers will become rough during production, the CGL produces outer coils first in a schedule. High surface requirement is also the reason behind that any outer coils can not be wider that its precedent coils to avoid edge marks on the coil surface. In order to ensure the quality of outer coils, when processing outer coils, the CGL needs to be examined and tuned from time to time. During the examination and tune, the production status of CGL may fluctuate, which influences the production quality. To reconcile this contradiction, an inner coil is needed to be produced after at most a specified number of outer coils are produced continuously. While the inner coil is produced, the examination and tune are performed. This is the most important feature of the CGLSP distinguished from other ones. A CGL schedule is shown in Fig. 1, in which each dark-colored bar corresponds to an outer coil and each light-colored one to an inner coil. A CGL schedule consists of two parts as shown in Fig. 1, outer coil part (OP) and inner coils part (IP). The outer coil part lies in the foreside of the schedule where inner coils are inserted. In outer coil part, inner coils are not allowed to be produced continuously. That is to say, there is at most one inner coil between two outer coils in OP. Because production makes rollers rough, the fewer inner coils are inserted between outer coils, the better the schedule is. Like most production lines in steel industry, the CGL has the constraints regarding maximum allowable jump of width and thickness between adjacent coils, and as small as possible the total jumps are expected. Besides constraints about width and thickness, jump on annealing temperature and thickness of 


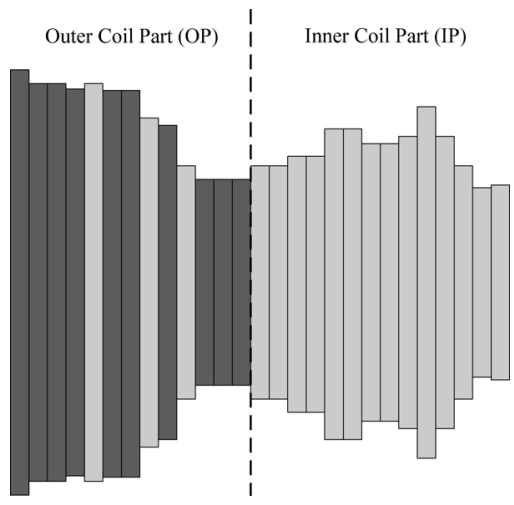

Fig. 1. A schedule of the continuous galvanizing line.

zinc coat between adjacent coils also have to be taken into consideration when the CGL schedules are made. The more changes happen on annealing temperature, the more energy are consumed, and frequently adjusting the equipment also expends much cost. For technical reasons, coils with specified post-processing can not be processed adjacently. We say two coils are incompatible if they can not be processed adjacently due to their post-processing or the excessive jumps.

The production mode in the plant is that the sales department releases customer orders to the manufacture department every week and the manufacture department organizes production according to the orders. The coils of all the orders received by the manufacturing department need regularly to be arranged a production sequence in advance which is given by a predictive schedule. This predictive schedule gives each coil an expected time when it is released to the CGL inventory. The given expected release time will guide the production scheduling arrangement at upstream operations of the CGL to meet the required time of coils at the CGL. Thus proper predictive schedule can efficiently coordinate the production between CGL operation and the upstream operations and may improve machine unitization. Because all coils are required to be scheduled during making a predictive scheduling and some coils may be incompatible, it is possible that no feasible schedule can be obtained. For infeasible situation, some virtual customer orders consisting of transition coils are introduced to construct a feasible schedule. The transition coils, even though belonging to virtual (open) orders, still are required to produce and supply to smooth CGL production. The production of the transition coils will result in additional cost. The holding cost will also occur as transition coils are stored for a long time until matched out by a practical order. Thus, the number of transition coils used should be considered in the objective function of the CGLSP.

According to the above description and analysis, the objective of the CGLSP is to minimize the following terms.

1) the total changeover cost between adjacent coils.

2) the number of inner coils inserted into outer coil part.

3) the number of transition coils that are absent but necessary to construct a feasible schedule.

\section{Model of the CGLSP}

By the above description, an integer programming model based is constructed. One of the objectives, the minimization of the number of transition coils in the model is converted to the minimization of the number of pairs of adjacent incompatible coils, because transition coils are needed only when two incompatible coils are adjacent produced in schedules.

\subsection{Variables and Notations}

Notations:

$N$ : The set of all coils, $N=\{0,1,2, \ldots, n\}$, where 0 denotes a dummy coil.

$O$ : The set of outer coils.

$I$ : The set of inner coils, $I \cup O=N, I \cap O=\varnothing$, where, and the dummy coil $0 \in I$.

$L O$ : The maximum allowable number of outer coils processed continuously.

$w d_{i}$ : The width of coil $i$.

$c_{i j}$ : The total changeover cost, covering the changeover cost of width, thickness, zinc coat thickness and annealing temperature, between coils $i$ and $j$.

$B_{i j}$ : The notation denotes whether coils $i$ and $j$ are compatible. If coils $i$ and $j$ are compatible, $B_{i j}=1$, otherwise $B_{i j}=0$.

$I C$ : The penalty of inserting an inner coil between outer coils.

$U C$ : The penalty of producing a transition coil.

Decision variables:

$x_{i j}= \begin{cases}1, & \text { if coil } j \text { is processed immediately after coil } i, \\ 0, & \text { otherwise } .\end{cases}$

Auxiliary variables:

$d_{i}$ : The position of coil $i$ in schedule, where $d_{0}=0$.

$o_{i}$ : The position of coil $i$ in the sub-sequence of outer coils produced continuously.

\subsection{Model}

Using the above notation, the CGLSP problem can be formulated as the following integer programming model:

Minimize

$$
\sum_{i \in N} \sum_{j \in N} B_{i j} c_{i j} x_{i j}+I C \sum_{i \in I \backslash\{0\}} \sum_{j \in O} x_{i j}+U C \sum_{i \in N} \sum_{j \in N}\left(1-B_{i j}\right) x_{i j}
$$

subject to

$$
\begin{aligned}
& \sum_{j \in N \backslash\{i\}} x_{i j}=1, \quad i \in N \\
& \sum_{i \in N \backslash\{j\}} x_{i j}=1, \quad j \in N \\
& \sum_{i, j \in S} x_{i j} \leq|S|-1, \quad \forall S \subseteq N \backslash\{0\}, 2 \leq|S| \\
& o_{i}=0, \quad i \in I \\
& o_{j} \geq o_{i}+1-\left(1-x_{i j}\right) L O, \quad i, j \in O, i \neq j . \\
& 1 \leq o_{j} \leq L O, \quad j \in O \\
& \left.x_{i j}=0, \quad j \in O, i \in M \backslash j\right\}, w d_{i}<w d_{j} . .
\end{aligned}
$$




$$
\begin{gathered}
x_{i j}+x_{j k} \leq 1, \quad i, k \in O, \quad j \in I, \quad w d_{i}<w d_{k} . \\
\sum_{i \in I \backslash\{j\}} x_{i j}+\sum_{k \in O} x_{j k} \leq 1, \quad j \in I \backslash\{0\} \ldots
\end{gathered}
$$

There are three terms in the objective function, which represent the total changeover cost between coils, the penalty of inner coils inserted at outer coil part, and the penalty of pairs of adjacent incompatible coils. Constraints (2) and (3) ensure that a coil can be processed once and only once. Constraint (4) is a popular constraint in models, which is to eliminate sub-cycles. However the number of inequalities in constraint (4) is exponential of $n$. In order to solve the model by using CPLEX 11.0, we transform the constraint into the following,

$$
\begin{array}{r}
d_{j} \geq\left(d_{i}+1\right)-\left(1-x_{i j}\right) n, \quad i \in N, j \in N \backslash\{0\} \\
d_{i} \leq n, \quad i \in N \ldots \ldots \ldots \ldots \ldots \ldots \ldots \ldots \ldots \ldots
\end{array}
$$

Constraints (5), (6) and (7) guarantee the number of outer coils produced continuously does not exceed the upper bound $L O$. Constraints (8) and (9) make sure that any outer coil is not wider than its precedent coils. The constraint (10) means that inner coils can not be produced continuously in the outer coil part.

\section{Initial Solution of TS}

The CGLSP is a generalization of TSP. As TSP is a NPhard problem and the CGLSP is more complicated than TSP with practical constraints, the CGLSP is NP-hard and intractable. For a NP-hard problem, it is not practical to obtain an optimal solution within reasonable computation time in many cases. We develop a TS based heuristic to get a near-optimal solution of the CGLSP in short computation time. Tabu search was proposed by Glover ${ }^{11)}$ in 1986 and by Hansen ${ }^{12)}$ in 1986 and has been proven to be an effective method to solve complicated combinational optimization problems arising from real industry.

A tabu search starts with an initial solution obtained by a heuristic. In this section, we describe a heuristic generating an initial solution.

The difficulty of the CGLSP follows the fact that it includes two sub-problems to solve: the sequencing problem of outer coils with the insertion of some inner coils and the sequencing problem of the rest inner coils. The interrelation of two sub-problems is obvious and hard to deal with. The heuristic of generating initial solutions consists of three sub-routines, sequencing outer coils, insertion inner coils and sequencing left inner coils.

\subsection{Sequencing Outer Coils}

Due to the constraint that any outer coil can not be wider than its precedent coils, it is quite easy to sequence all outer coils when none of coils has the same width. We sequence outer coils by the decreasing order of width first. If none of coils has the same width, the sub-routine of generating the sequence of outer coils terminates; otherwise a dynamic programming method is applied to sequence coils with the same width $W D$. Sequencing these coils can be regarded as a problem to find a shortest Hamiltonian path with the given start point and end point with visiting all nodes in a transition graph $G=(V, E)$. $V$ consists of coils, 1 to $m$, with the same width, a start point, 0 , representing a coil which is the narrowest in all outer coils wider than $W D$, and an end point, $m+1$, representing a coil which is the widest in all outer coils narrower that $W D$. The arc set $E$ consists of arcs whose endpoints represent coils processed adjacently and each arc is associated with a weight defined by the changeover cost between its endpoints. If coil 0 or $m+1$ does not exist, a dummy coil is added and the weights of arcs linking the dummy coil to other coils are equal to 0 .

Dynamic programming is not an effective method to solve TSP because the number of status increases in exponential growth as the problem size increases. However, in the practical CGLSP the number of coils with the same width is not large, so dynamic programming can find an optimal solution very fast.

\subsection{Inserting Inner Coils}

Each position between two outer coils is a potential position to insert an inner coil. One of the objectives of the problem is to insert inner coils as few as possible and the number of outer coils between two inner coils does not exceed $L O$. A nature idea is to insert an inner coil after every $L O$ outer coils. But the scheduling problem also minimizes the total changeover cost between coils, so more effective method is needed.

This sub-routine is to insert inner coils between outer coils. We regard this sub-routine as an assignment problem between positions and inner coils and employ Hungarian method to solve it. To get the tradeoff between the objectives, the minimization of the number of inserted inner coils and the minimization of the total changeover cost, the assignment cost is the sum of these two parts. The first part is independent upon the inserted coil and only involves the position itself. We use $p$ to index all the positions and position $p$ is the one after $p$-th outer coil. Obviously $p$ ranges from 0 to $|O|-1$. Let ${ }_{p}^{I C}$ denote the first part of assignment cost. The dummy coil 0 is deemed to be an inner coil, and it is an unavoidable inner coil though its cost does not be taken into the objective function. If two outer coils processed adjacently are incompatible, the position between these two coils must be occupied by an inner coil. Thus an unavoidable inner coil exists at the position between two incompatible coils. For each position $p, P U_{p}$ and $F U_{p}$ denote the position of its precedent and following unavoidable inner coils nearest to $p$. Thus $c_{p}^{I C}$ is calculated as follows,

$$
c_{p}^{I C}=\left\{\begin{array}{l}
0, \quad \begin{array}{l}
\text { if } p \text { is the position of an unavoidable } \\
\text { inner coil },
\end{array} \\
I C \cdot \min \left\{\frac{L O-\operatorname{Mod}\left(\left(p-P U_{p}\right), L O\right)}{L O}\right. \\
\left.\frac{L O-\operatorname{Mod}\left(\left(F U_{p}-p\right), L O\right)}{L O}\right\} \\
\text { otherwise }
\end{array}\right.
$$

where the second term in $\min \{\cdot, \cdot\}$ is equal to 1 if $F U_{p}$ does not exist for $p$.

We adopt two ways, respectively named $\mathrm{C} 1$ and $\mathrm{C} 2$ to 
calculate the second part of assignment cost for each pair of inner coil and position. A sequence of all inner coils is generated by the nearest neighbor heuristic $(\mathrm{NNH})$. The sequence is started with the narrowest outer coil, and then the inner coil with the smallest changeover cost with the last coil in the sequence is added, and so on. For position and inner coil, $\operatorname{prev}(\cdot)$ and $\operatorname{next}(\cdot)$ are defined to denote its previous coil and its next coil. With these notations, two ways to calculate the second part of assignment cost between position $p$ and inner coil $i$ is defined as follows,

$$
\begin{aligned}
\mathrm{C} 1: \quad \operatorname{AssignmentCost}(p, i)= & c_{\text {prev }(p), i}+c_{i, \operatorname{next}(p)} \\
\mathrm{C} 2: \quad \operatorname{AssignmentCost}(p, i)= & \left.c_{\text {prev }(p), i}+c_{i, \operatorname{next}(p)}-c_{\operatorname{prev}(i), i}-c_{i, \operatorname{next}(i)}\right) \\
& +\left(c_{\operatorname{prev}(i), \operatorname{next}(i)}\right.
\end{aligned}
$$

In way $\mathrm{C} 1$, only the cost brought by insertion is considered, while in way $\mathrm{C} 2$, the possible increase cost resulted from the unavailability of the inner coil when sequencing inner coils is taken into consideration. The classical Hungarian method deals with the assignment problem with the same number of jobs and people, but in our problem the number of inner coils is always larger than the number of positions. Dummy positions are made and the number of these dummy positions is equal to the difference between the numbers of real position and inner coils. The cost of assigning an inner coil to a dummy position is $-M$ which is much smaller than other assignment cost.

Though we get a feasible sub-sequence here, too many inner coils are inserted. In order to reduce the number of inserted inner coils, a simply heuristic is applied as follows.

Calculate the insert cost for each inserted inner coil $i$ as,

$$
I C_{i}=I C+c_{\text {prev }(i), i}+c_{i, \text { next }(i)}-c_{\text {prev }(i), \operatorname{next}(i)}
$$

Sort all inserted inner coils by decreasing order of $I C_{i}$. Try to delete the inserted coils one by one from the sub-sequence. If the obtained sub-sequence is feasible, perform the deletion; otherwise try to delete the next coil.

\subsection{Sequencing Inner Coils}

The nearest neighbor heuristic (NNH) is employed to sequence the left inner coils. NNH is not a very good method for TSP or VRP though with a very nature thought. The reason behind this is that TSP or VRP needs a tour but not a path. When NNH is used to solve TSP, the arc connecting the depot and the last customer always contributes the most in traveling distance. Although we regard the CGLSP as a generalization of TSP, it is the dummy coil that presents the start and the end of schedules, so the cost between each coil and the dummy coil is 0 . Thus the disadvantage of $\mathrm{NNH}$ does not exist in our problem. NNH is also used to solve Open VRP similar to our problem in Ref. 10). The proce- dure of NNH is quite simple and is the same to the procedure described above.

\section{Major Components of Tabu Search for CGLSP}

The TS is based on a neighborhood or local search technique and its principle is to make the recent moves in tabu status to avoid being wrapped at local optimum.

\subsection{Neighborhoods}

Generally speaking, the neighbor solution $S^{\prime}$ is obtained by applying a move $m$ to the base solution $S$. Neighborhood $N_{x}(S)$ is the set of neighbor solution $S^{\prime}$ obtained by applying move $m_{x}$ on the solution $S$. In the paper we use five kinds of moves, 2-Opt, path insert, path swap, coil exchange and coil swap, to construct the five neighborhoods. The first three moves are also used in Ref. 8), but due to the different characters between CGLSPs, we further design other two moves to meet the special features of our CGLSP. Here we introduce these five kinds of moves.

The 2-Opt neighborhood consists of new solutions obtained by removing two arcs and adding two new arcs. The 2-Opt move is shown in Fig. 2. The nature of 2-Opt is to reverse a sub-sequence in the schedule.

The path insert move is to remove three arcs and to add three new arcs. The move is shown in Fig. 3. There are two modes of this move, normal path insert and reverse path insert. In the figure the path from $a$ to $b$ is reversed and inserted between $c$ and next $(c)$, so it is a reverse path insert. If $\operatorname{arcs}(c, a)$ and $(b, n \operatorname{ext}(c))$ are added, it is a normal path insert. A special case is that, when $a$ and $b$ are the same coil, the path insert move becomes the coil relocation move.

The path swap move, as shown in Fig. 4, is to swap two paths in the schedule. Because two paths are involved in the
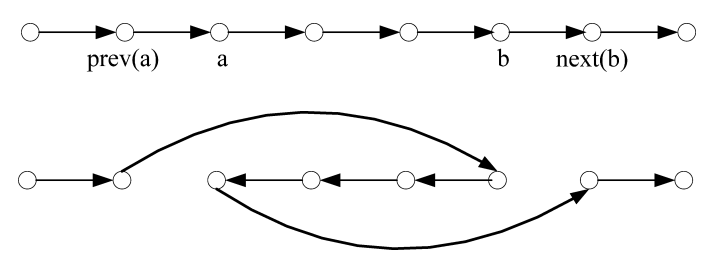

Fig. 2. 2-Opt move.

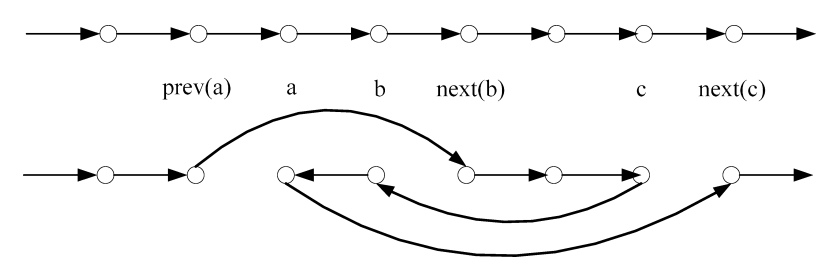

Fig. 3. Path insert move.
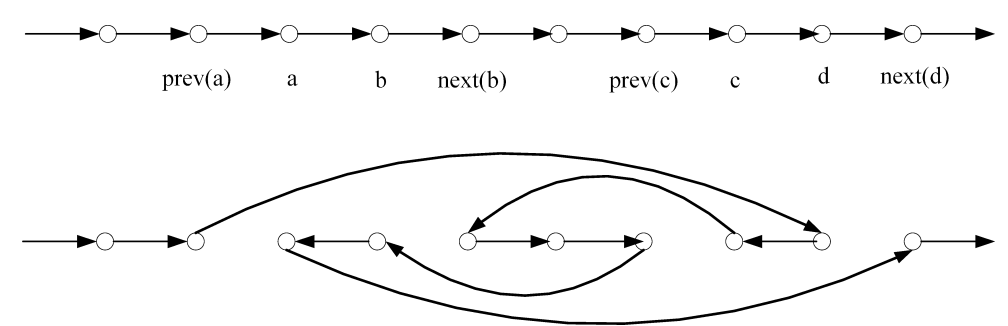

Fig. 4. Path swap move. 
path swap move, similar to the path insert, the path swap has four modes, (normal, normal), (normal, reverse), (reverse, normal) and (reverse, reverse). The path swap removes and adds four arcs respectively. For a schedule with $n$ arcs, its complexity is $O\left(n^{4}\right)$. It is very time consuming to search such a neighborhood. To reduce the neighborhood size, the length (the number of arcs in path) of swapped path is limited under three. When the lengths of both paths are equal to 0 , the path swap becomes the coil swap.

The well-known $k$-opt move for the TSP replaces $k$ arcs in the tour by any other $k$ arcs so that a new tour can be constructed. From this point, the path insert move and path swap move are similar to 3-opt and 4-opt moves. But some evident differences between these moves should be noticed, and we here use 3-opt move and the path insert move as the example to illustrate the differences. For 3-opt move, removing three arcs from a tour obtains three sub-paths, and these three sub-paths can be reversed and the sequence among them can be re-arranged.

In our research, coils are arranged in a path rather than a tour. Though a dummy coil is added in the CGLSP model, our algorithm orients to the practical path express. For the path insert move, removing three arcs from a path obtains four sub-paths, and the first and last sub-paths are fixed, which are neither able to be moved nor to be reversed. Only the middle two sub-paths can be moved. In path insert move, the second sub-path is inserted after the third subpath, and it can be reversed, while the third sub-path can not be reversed. The differences between the path swap move and 4-opt move is similar to that between the path insert move and 3-opt move.

The above three kinds of moves are only applied to inner coil part. The reason is that the schedule in outer coil part is very constrained and most of these moves obtain infeasible schedules.

The exchange move is that, relocate an inner coil from OP to IP and place another inner coil to the position. In Fig. 5 coil $a$ is relocated after coil $b$ and coil $c$ is placed to the position where $a$ was. Each exchange move involves the replace of five arcs. If coil $c$ is narrower than $\operatorname{next}(a)$, the move can not be applied because of the width constraint.

The coil swap move is to swap the positions of outer coils with the same width.

To reduce the computing, when we evaluate a move, only the increment about the move is calculated. For different moves, calculate the increment $C(m)$ as follows.

\section{2-Opt: $\quad \Delta C(m)=\left(c_{\text {prev }(a), b}+c_{a, \operatorname{next}(b)}\right)-\left(c_{\operatorname{prev}(a), a}+c_{b, \operatorname{next}(b)}\right)$}

Path insert:

$$
\Delta C(m)=\left\{\begin{array}{c}
\left(c_{c, a}+c_{b, \operatorname{next}(c)}+c_{\operatorname{prev}(a), \operatorname{next}(b)}\right) \\
-\left(c_{\operatorname{prev}(a), a}+c_{b, \operatorname{next}(b)}+c_{c, \operatorname{next}(c)}\right) \\
\operatorname{normal} \operatorname{move} \\
\left(c_{c, b}+c_{a, \operatorname{next}(c)}+c_{\operatorname{prev}(a), \operatorname{next}(b)}\right) \\
-\left(c_{\operatorname{prev}(a), a}+c_{b, \operatorname{next}(b)}+c_{c, \operatorname{next}(c)}\right) \\
\text { reverse move }
\end{array}\right.
$$

Path swap:

$$
\Delta C(m)=\left\{\begin{array}{c}
\left(c_{\operatorname{prev}(a), c}+c_{d, \operatorname{next}(b)}+c_{\operatorname{prev}(c), a}+c_{b, \operatorname{next}(d)}\right) \\
-\left(c_{\operatorname{prev}(a), a}+c_{b, \operatorname{next}(b)}+c_{\operatorname{prev}(c), c}+c_{d, \operatorname{next}(d)}\right), \\
(\operatorname{normal}, \operatorname{normal}), \\
\left(c_{\operatorname{prev}(a), c}+c_{d, \operatorname{next}(b)}+c_{\operatorname{prev}(c), b}+c_{a, \operatorname{next}(d)}\right) \\
-\left(c_{\operatorname{prev}(a), a}+c_{b, \operatorname{next}(b)}+c_{\operatorname{prev}(c), c}+c_{d, \operatorname{next}(d)}\right), \\
(\operatorname{reverse}, \operatorname{normal}), \\
\left(c_{\operatorname{prev}(a), d}+c_{c, \operatorname{next}(b)}+c_{\operatorname{prev}(c), b}+c_{a, \operatorname{next}(d)}\right) \\
-\left(c_{\operatorname{prev}(a), a}+c_{b, \operatorname{next}(b)}+c_{\operatorname{prev}(c), c}+c_{d, \operatorname{next}(d)}\right), \\
(\operatorname{normal}, \operatorname{reverse}), \\
\left(c_{\operatorname{prev}(a), d}+c_{c, \operatorname{next}(b)}+c_{\operatorname{prev}(c), b}+c_{a, \operatorname{next}(d)}\right) \\
-\left(c_{\operatorname{prev}(a), a}+c_{b, \operatorname{next}(b)}+c_{\operatorname{prev}(c), c}+c_{d, \operatorname{next}(d)}\right), \\
(\operatorname{reverse}, \operatorname{reverse})
\end{array}\right.
$$

Coil Exchange:

$$
\begin{aligned}
\Delta C(m)= & \left(c_{\operatorname{prev}(a), c}+c_{c, \operatorname{next}(a)}+c_{b, a}+c_{a, \operatorname{next}(b)}+c_{\operatorname{prev}(c), \operatorname{next}(c)}\right) \\
& -\left(c_{\operatorname{prev}(a), a}+c_{a, \operatorname{next}(a)}+c_{b, \operatorname{next}(b)}+c_{\operatorname{prev}(c), c}+c_{c, \operatorname{next}(b)}\right)
\end{aligned}
$$

Coil Swap:

$$
\begin{aligned}
\Delta C(m)= & \left(c_{\text {prev }(a), b}+c_{b, \operatorname{next}(a)}+c_{\text {prev }(b), a}+c_{a, \text { next }(b)}\right) \\
& -\left(c_{\operatorname{prev}(a), a}+c_{a, \operatorname{next}(a)}+c_{b, \operatorname{next}(b)}+c_{b, \operatorname{next}(b)}\right)
\end{aligned}
$$

\subsection{Tabu List and Stopping Criteria}

Tabu list is a section of memory where the reverse moves of recent $\theta$ iterations are kept, and $\theta$ is a predetermined number. In most cases, the tabu list is cyclic and its size is $\theta$. However the move in our heuristic always involves at least more than two arcs and the number of involved arcs is various, so the classic cyclic tabu list is inconvenient in our implementation. In our algorithm a quadrate memory with size $n \times n$ is proposed to kept reverse moves. The element indexed by $[i][j]$ denote the status of the arc linking coils $i$

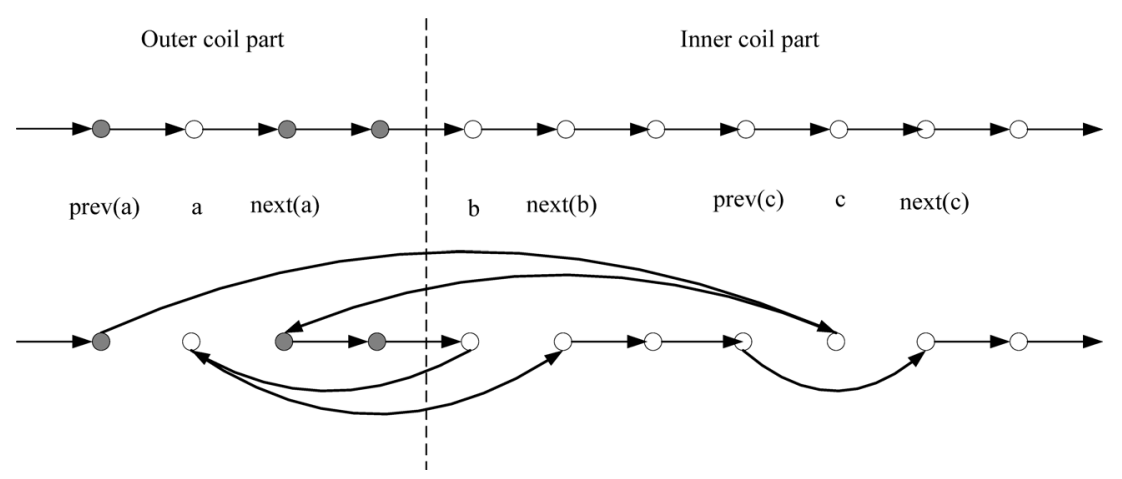

Fig. 5. Coil exchange move. 


\begin{tabular}{|l|l|l|l|l|l|}
\hline 0 & 0 & 0 & 0 & 0 & 0 \\
\hline 0 & 0 & 0 & 0 & 0 & 0 \\
\hline 0 & 0 & 0 & 0 & 0 & 0 \\
\hline 0 & 0 & 0 & 0 & 0 & 0 \\
\hline 0 & 0 & 0 & 0 & 0 & 0 \\
\hline 0 & 0 & 0 & 0 & 0 & 0 \\
\hline
\end{tabular}

(a)

\begin{tabular}{|l|c|c|c|c|c|}
\hline-1 & -1 & 3 & -1 & -1 & -1 \\
\hline-1 & -1 & -1 & -1 & -1 & -1 \\
\hline-1 & -1 & -1 & -1 & -1 & -1 \\
\hline-1 & -1 & -1 & -1 & 3 & -1 \\
\hline-1 & -1 & -1 & -1 & -1 & -1 \\
\hline-1 & -1 & -1 & -1 & -1 & -1 \\
\hline
\end{tabular}

\begin{tabular}{|c|c|c|c|c|c|}
\hline-2 & 3 & 2 & -2 & -2 & -2 \\
\hline-2 & -2 & -2 & -2 & -2 & -2 \\
\hline-2 & -2 & -2 & -2 & -2 & -2 \\
\hline-2 & -2 & -2 & -2 & 2 & -2 \\
\hline-2 & -2 & -2 & -2 & -2 & 3 \\
\hline-2 & -2 & -2 & -2 & -2 & -2 \\
\hline
\end{tabular}

(c)

Fig. 6. An example of our tabu list.

and $j$. Each element in the memory is initialized as 0 .

Five kinds of moves can be categorized into path oriented move and coil oriented move. 2-Opt, path insert and path swap are path oriented move, and the other two are coil oriented move. For path oriented move, all arcs removed are added into tabu list, and for coil oriented move, only arcs linking moved coils and their immediately precedent moves are added. If we perform the move in which $k$ arcs, $\left(i_{1}, j_{1}\right),\left(i_{2}, j_{2}\right), \ldots,\left(i_{k}, j_{k}\right)$, are to be added into the tabu list in an iteration, after the iteration we subtract 1 from each element and then let elements $\left[i_{1}\right]\left[j_{1}\right],\left[i_{2}\right]\left[j_{2}\right], \ldots,\left[i_{k}\right]\left[j_{k}\right]$ be equal to $\theta$ which means the tabu tenure. The ways to judge whether a move is in tabu status or not are different for path oriented move and coil oriented move. For a path oriented move, if the values of two new arcs linking to any involved path in the move are both positive, the move is in tabu status. For example, in Fig. 4 path $a$ to $b$ is moved between $\operatorname{prev}(c)$ and $n \operatorname{ext}(d)$. If the values of elements $[\operatorname{prev}(c)][b]$ and $[a][n \operatorname{ext}(d)]$ in memory are positive, the move in Fig. 4 is in tabu status. For a coil oriented move, if any involved coil in the move is placed after another coil and the element value of the arc between two coils is positive, the move is in tabu status. Figure 6 shows the status of our tabu list with $\theta=3$. The initial status is in (a); in (b) arcs $(1,3)$ and $(4,5)$ are added in the tabu list, so the corresponding element value are set by 3 ; in (c) all elements are subtracted by 1 and some elements are set by 3 . An arc added in the tabu list is free until its corresponding element is not positive.

Two common stopping criteria have been chosen to terminate the tabu search. They consist of the maximum total number of iterations (MaxIter) and the maximum number of iterations without improvement of the best known solution (MaxIterWI). The tabu search is terminated when either of these two criteria is attained.

\subsection{Additional Tabu Search Features}

Besides the standard tabu search feature, additional improving strategies are used to make the tabu search more effective.

\subsubsection{Intensification Search}

A simple idea is that the larger the neighborhood is, the better schedule is obtained. But searching a larger neighborhood consumes more time. To tradeoff the contradiction between neighborhood size and computation time, the intensification search is used. The nature of the intensification search is to intensify the search in a seemingly promising region or to periodically enlarge the neighborhood size.

Variable depth search (VDS) has been focused on in recent years. In each iteration, VDS compounds some primary moves to search larger domain. Because the large domain search ability of VDS meets the requirement of intensification search strategy, we implement filter-and-fan (F\&F) method which is based on variable depth thought in the intensification search. F\&F proceeds at most $M A X L E V E L$ levels and returns the best found solution during the search. Considering the intensification search starts with solution $S$, the filter-and-fan method refereeing ${ }^{13)}$ is as follows.

Step 1. Empty the solution sets $S^{(1)}, S^{(2)}, \ldots, S^{(\text {MaxLvl) }}, S^{\text {pool }}$ and let $l e l=0, S^{(0)}=\{S\}$.

Step 2. For each solution in $S^{(l v l)}$, find [ $\left.\eta_{1} / 5\right]$ best solutions in each of the described five neighborhoods and keep these solutions in the set $S^{\text {pool }}$.

Step 3. Select best $\eta_{2}$ solutions in $S^{\text {pool }}$ and put these solution in $S^{(l v l+1)}$. Let $l v l=l v l+1$. If $l v l<M A X L E V E L$, go to step 2, otherwise return the best solution in the union of $S^{(1)}, S^{(2)}, \ldots, S^{(M A X L E V E L)}$ and quit the method.

We apply the intensification search every [MaxIterWI/3] iterations. If the new best known schedule is found in the recent [MaxIterWI/3] iterations, the intensification search is applied upon this schedule; if no new best known schedule is found, the local search is applied upon the current base schedule. If the new schedule found in intensification search is better than the best known schedule, update the best known schedule and continue the tabu search; otherwise continue the tabu search with the base schedule before intensification search started.

\subsubsection{Diversification Search}

The intention of the diversification search is to make our search work in more extensive neighborhood, especially the domain where is hard to arrive for the normal local search.

In our heuristic the diversification search is to select the best schedule in the set of schedules obtained by applying operations named shift on the best known schedule. The best new schedule found in the diversification search is used to reinitialize the tabu search. The diversification search is applied when no new best known schedule is found in recent [MaxIterWI/3] iterations.

The shift operation is composed of an uncertain number of swap or insert move. The first step of shift operation is to shift an inner coil in outer coil part either forward or backward. The direction to the starts of schedules is forward, and backward is to the ends of schedules. A single shift move can be regarded as a swap move with its adjacent outer coils. The shift may result in that the number of outer coils processed continuously exceeds the max number limit $L O$. When the violation arises, two repair strategies are applied and get different schedules. The better schedule is accepted as the result of this shift operation.

The first repair strategy is to insert another inner coil into 
the excessive long sub-sequence. The insertion cost is equal to the assignment cost C2: AssignmentCost $(p, i)$. The coil and the position with the minimum assignment cost are selected. And the second repair strategy is as follows.

Step 1. Let Si denote the shifted inner coil and Nsi denote the next shifted inner coil in the repair procedure.

Step 2. If $\mathrm{Si}$ is shifted forward in the schedule, let $N s i$ be its nearest following inner coil; otherwise let Nsi be its nearest precedent inner coil.

Step 3. If $N s i$ is an unavoidable inner coil, the repair strategy fails. If the shift direction is forward, and Nsi is narrower than its immediately precedent outer coil, the repair strategy fails, too.

Step 4. Shift Nsi in the direction in that $S i$ is shifted.

Step 5. If the schedule is feasible, quit the procedure; otherwise set $\mathrm{Si}=N s i$, and go to Step 2 .

It is worth mentioning that if the first shift move of the shift operation results in that two inner coils are adjacent in outer coil part, the operation is forbidden. The new schedule of shift operation is difficult to forecast, so no equations is available to calculate the increment of the operation. To evaluate a shift operation, the objective function value needs to be recalculated.

\subsubsection{Path Relinking}

Path relinking is frequently used in recent years, especially in tabu search and scatter search. Considering two schedules $S_{1}$ and $S_{2}$, path relinking is to find a series of schedules which compose a path linking $S_{1}$ and $S_{2}$. Between each two adjacent schedules in the path there exists a slight modification.

Without losing general, we let schedule $S_{1}$ be the start of the path and $S_{2}$ be the end. Denotation $S(i)$ means the $i$-th coil in the schedule $S$. The path relinking procedure can be summarized as follows.

Step 1. Let $i=1, S^{P R}=S_{1}$.

Step 2. If $S^{P R}(i)=S_{2}(i), i=i+1$ and repeat step 2 .

Step 3. Find $S^{P R}(k)=S_{2}(i)$, and swap coils $S^{P R}(i)$ and $S^{P R}(k)$.

Step 4. If $S^{P R}$ is feasible and better than the best known schedule, than update the best known schedule with $S^{P R}$.
Step 5. If $i \geq n$, quit the procedure; otherwise $i=i+1$, and go to step 2 .

The path relinking is applied to the pair of the best known schedule and the current schedule after each iteration.

\section{Computational Results}

This section describes the computational results used to evaluate the heuristic. The heuristic was programmed in $\mathrm{C}++$ and runs on a personal computer with 3.0G Pentium IV CPU and 1024 Mbytes of RAM. CPLEX 11.0 Optimizer runs on a personal computer with Core E6550 CPU and 4096 Mbytes of RAM.

\subsection{Test Problems}

We test our heuristic on instances of four sizes that we randomly generated to simulate actual production data in a steel plant. Four instance sizes are $n=20,30,50,100$, and for all size instances the changeover cost is uniformly from $[0,150]$. The parameter $I R$ is used to initialize the incompatible relationship between coils. When generating changeover cost, each pair of coils may be incompatible in probability of $I R \%$. And $L O$ is various for different sizes. The instances of four sizes are specified in Table 1. The reason why only three instances with 100 coils are generated is that solving such instances takes much time, and the significant advantage of our heuristic can be observed on even though only three instances and is convincing.

\subsection{Numerical Experiments}

We first test the influence of the initial solution with different ways of generating assignment cost $\mathrm{C} 1$ and $\mathrm{C} 2$. In Table 2, the average initial solution values of different ways are shown in columns Obj. value respectively, and the gaps between the initial solution and the optimal solution or best found solution obtained by CPLEX 11.0 are in the columns Gap (\%). Because both computation times of two ways are small, the computation time is not presented in the table. From Table 2 we can conclude that initial solutions generated in $\mathrm{C} 2$ are better than $\mathrm{C} 1$, though this does not happen

Table 1. Test data.

\begin{tabular}{lcccc}
\hline Instance Size $(n)$ & 20 & 30 & 50 & 100 \\
\hline Number of instances & 10 & 10 & 5 & 3 \\
$O L$ & 2 & 2 & 3 & 4 \\
$I R(\%)$ & 10 & 10 & 20 & 20 \\
the proportion of outer coils & 20 & 20 & 20 & 20 \\
\hline
\end{tabular}

Table 2. Comparison between initial solutions generated by $\mathrm{C} 1$ and $\mathrm{C} 2$.

\begin{tabular}{|c|c|c|c|c|c|c|}
\hline \multirow{2}{*}{$n$} & \multirow{2}{*}{$\begin{array}{l}\text { Number of } \\
\text { instances }\end{array}$} & \multirow{2}{*}{$\begin{array}{c}\text { CPLEX } \\
11.0\end{array}$} & \multicolumn{2}{|c|}{$\mathrm{C} 1$} & \multicolumn{2}{|c|}{$\mathrm{C} 2$} \\
\hline & & & Obj. Value & Gap $(\%)$ & Obj. Value & Gap(\%) \\
\hline 20 & 10 & 574.8 & 632.4 & 10.10 & 609.3 & 6.09 \\
\hline 30 & 10 & 883.1 & 978.3 & 10.76 & 962.5 & 8.95 \\
\hline 50 & 5 & 1236 & 1465 & 18.53 & 1444.5 & 16.83 \\
\hline 100 & 3 & 2459 & 2860 & 16.30 & 2830 & 15.08 \\
\hline
\end{tabular}


Table 3. Comparison of using different tabu tenure.

\begin{tabular}{|c|c|c|c|c|c|c|}
\hline \multirow{2}{*}{ Instance $(n)$} & \multicolumn{2}{|c|}{$n / 2$} & \multicolumn{2}{|c|}{$n / 3$} & \multicolumn{2}{|c|}{$n / 4$} \\
\hline & Obj. Value & Time(s) & Obj. Value & Time(s) & Obj.Value & Time(s) \\
\hline $1(100)$ & 2179 & 24.61 & 2168 & 24.05 & 2168 & 25.27 \\
\hline $2(100)$ & 2129 & 9.48 & 2119 & 9.44 & 2119 & 9.14 \\
\hline $3(100)$ & 2028 & 15.25 & 2028 & 24.92 & 2029 & 19.39 \\
\hline $4(50)$ & 1206 & 6.15 & 1206 & 7.59 & 1206 & 7.82 \\
\hline $5(50)$ & 1175 & 7.15 & 1168 & 8.31 & 1173 & 7.48 \\
\hline $6(50)$ & 1231 & 2.46 & 1231 & 3.27 & 1231 & 2.88 \\
\hline $7(50)$ & 1324 & 7.63 & 1324 & 12.30 & 1324 & 10.1 \\
\hline $8(50)$ & 1169 & 8.31 & 1157 & 9.13 & 1157 & 8.41 \\
\hline Avg. & 1555.13 & 10.13 & 1550.13 & 12.38 & 1550.88 & 11.31 \\
\hline
\end{tabular}

Table 4. Results of Tabu variants for solving instances with 100 coils.

\begin{tabular}{|c|c|c|c|c|c|c|c|c|}
\hline \multirow{2}{*}{$\begin{array}{c}\text { Instance } \\
(n)\end{array}$} & \multicolumn{2}{|c|}{ DS\&IS } & \multicolumn{2}{|c|}{ IS } & \multicolumn{2}{|c|}{ DS } & \multicolumn{2}{|c|}{ None } \\
\hline & Obj & Time & Obj & Time & Obj & Time & Obj & Time \\
\hline $1(100)$ & 2168 & 24.05 & 2205 & 4.20 & 2203 & 13.39 & 2208 & 5.88 \\
\hline $2(100)$ & 2119 & 9.44 & 2130 & 4.58 & 2130 & 7.73 & 2130 & 4.79 \\
\hline $3(100)$ & 2028 & 24.92 & 2063 & 5.73 & 2029 & 14.27 & 2063 & 5.78 \\
\hline Avg. & 2105 & 19.47 & 2132.67 & 4.84 & 2120.67 & 11.80 & 2133.67 & 5.48 \\
\hline
\end{tabular}

on every instance.

The comparison of using different tabu tenure $(n / 2, n / 3$, $n / 4$ ) is shown in Table 3. The experiments are performed on the eight instances with 50 and 100 coils. The tabu search starts with the initial solution generated in way $\mathrm{C} 2$. For each instance, MaxIter and MaxIterWI are 500 and 250 respectively. All other tests are all performed on this parameter configuration. In Table 3 the tabu search variant with $n / 2$ tabu tenure consumes the least computation time, but obtains the worst solution. The results obtained with $n / 3$ and $n / 4$ are not significant different. For six out of eight instances, the heuristics with $n / 3$ and $n / 4$ get the completely same objective values, and slight differences happen on only two instances. The heuristic variant with $n / 3$ consumes more time by $9.46 \%$ and obtains better solution by $0.048 \%$ than that with $n / 4$. Because of the better solution of $n / 3$, the tabu tenure is $n / 3$ in the following tests.

To compare the impact of intensification search and diversification search, the results of Tabu variants on instances with 100 coils are shown in Table 4. In the term DS\&IS, both two strategies are applies. The results of Tabu variant which applies solely IS or DS are in the terms IS and DS. Finally the results of elementary tabu search are shown in the term None. When being applied solely, the intensification search does not show obvious advantage, whereas the diversification search obtains better solutions than intensification search does. However the best solutions are always obtained by the variant with both two strategies, which consumes more computation time more than applying solely diversification search by about $65 \%$.

In Table 5, we evaluate the effectiveness of our tabu search heuristic by comparing with the solution obtained by the standard software package CPLEX 11.0. We indicate the value of the objective function and the computation time for both approaches. The computation time of the standard software is limited to $600 \mathrm{~min}$. In the case that the software obtains an optimal solution within the time limitation, the gap is equal to 0 ; otherwise a lower bound (LB) and the gap between the best found solution and the LB are given. The average gaps between our heuristic solution and optimal solution are in the term of Tabu in Table 5. Without optimal solutions, the lower bounds are used to calculate the gaps.

For all instances with 20 and 30 coils and four out of five instances with 50 coils, the software obtains optimal solutions. For the other instances, the software does not reach optimal solutions. Our tabu search heuristic obtains the optimal solutions in all instances on that the software obtains. For the instances that the software does not obtain optimal solutions, our heuristic gets better solutions and the average gap between the lower bound is smaller than $10 \%$, though the gap may be tighter if more time is given to CPLEX. However the computation time of the heuristic is much smaller than the software.

\section{Conclusion}

In this paper we have described the CGLSP arising in a steel company, which is much different to other CGLSPs having been published on some special features. We have formulated it as an integer programming model and developed a heuristic based on TS to solve it. The comparison 
ISIJ International, Vol. 49 (2009), No. 3

Table 5. Comparison between schedules obtained with CPLEX 11.0 and with tabu search.

\begin{tabular}{clcc}
\hline$n$ & & CPLEX 11.0 & Tabu \\
\hline 20 & Objective Value & 574.89 & 574.89 \\
& Lower Bound & 574.89 & - \\
& Gap (\%) & 0 & 0 \\
& Time (seconds) & 3.51 & 0.86 \\
30 & Objective Value & 883.10 & 833.10 \\
& Lower Bound & 883.10 & - \\
& Gap (\%) & 0 & 1.5 \\
& Time (seconds) & 167.43 & 1217.3 \\
50 & Objective Value & 1232.80 & - \\
& Lower Bound & 1203.20 & 1.17 \\
& Gap (\%) & 2.46 & 8.12 \\
& Time (seconds) & 11732 & 2105 \\
100 & Objective Value & 2347.00 & - \\
& Lower Bound & 1965.66 & 19.47 \\
\hline Gap (\%) & 19.40 & 36000 \\
Time (seconds) & &
\end{tabular}

between schedules obtained by our heuristic and CPLEX has been done and the computational results show that our heuristic is effective and can obtain high quality schedules. For the instances examined, when CPLEX obtains optimal solutions, the heuristic can also obtain optimal solutions. When CPLEX did not obtain optimal solutions, the heuristic can always obtain better solutions than CPLEX. For all instances, the computation time consumed by the heuristic is much less than that by CPLEX. In order to facilitate the practical application, embedding the heuristics, we developed the decision support system containing the schedule edit system which expresses schedules in graphic view and enables users to edit schedules generated automatically with drag and drop way.

\section{Acknowledgement}

This research is partly supported by National Natural Science Foundation for Distinguished Young Scholars of China (Grant No. 70425003), National 863 High-Tech Research and Development Program of China through approved No. 2006AA04Z174 and National Natural Science Foundation of China (Grant No. 60674084).

\section{REFERENCES}

1) H. S. Lee, S. S. Murthy, S. W. Haider and D. V. Morse: IBM J. Res. Dev., 40 (1996), No. 2, 231.

2) L. Lopze, M. W. Carter and M. Gendreau: Eur. J. Oper. Res., 106 (1998), No. 2, 317

3) P. Cowling, D. Ouelhadj and S. Petrovic: J. Intelligent Manufact., 14 (2003), No. 5, 457.

4) P. Cowling: Comput. Ind. Eng., 45 (2003), No. 2, 307.

5) L. X. Tang, J. Y. Liu, A. Y. Rong and Z. H. Yang: Euro. J. Oper. Res., 133 (2001), No. 1, 1.

6) S. Moon and A. N. Hrymak: Comput. Chem. Eng., 23 (1999), No. 9, 1193.

7) L. X. Tang and X. P. Wang: Int. J. Adv. Manufact. Technol., 35 (2008), No. 7, 633.

8) H. Okano, T. Morioka and K. Yoda: Research Report RT-0478, IBM Tokyo Research Laboratory, 1623-14 Shimo-tsuruma, Yamato-shi, Kanagawa-ken 242-8502, Japan, (2002).

9) V. V. Verdejo, M. A. P. Alarco and M. P. L. Sorli: Compu. Oper. Res., 36 (2009), No. 1, 280.

10) J. Brandao: Eur. J. Oper. Res., 157 (2004), No. 3, 552.

11) F. Glover: Comput. Oper. Res., 13 (1986), No. 5, 533.

12) P. Hansen: Proc. of Cong. on Numerical Methods in Combinatorial Optimization, Capri, Italy, (1986).

13) P. Geristorfer and C. Rego: Comput. Oper. Res., 33 (2006), No. 4, 2590. 